\title{
Etudiants en temps de confinement et au-delà
}

Elodie Charbonnier, Sarah Le Vigouroux et Aurélie Goncalves

UNIV. NIMES, APSY ${ }^{-\mathrm{V}}$, F-30021 Nîmes Cedex, France 
Presse Médicale Formation

Points essentiels :

- les confinements ont augmenté les symptomatologies anxieuses et dépressives des étudiants Français.

- Au-delà des confinements, la situation sanitaire a augmenté la présence de symptômes anxieux chez les étudiants.

- Il semble que les étudiants aient augmenté leur temps d'activité physique et sportive durant le premier confinement, mais que cela ne soit pas maintenu dans le temps, même lors du second confinement.

- La crise sanitaire a eu pour conséquence d'augmenter les comportements sédentaires.

- Il est important pour les universités d'accompagner les étudiants au-delà des apprentissages, dans le vécu émotionnel et leur mode de vie.

- Dans ce manuscrit, nous proposons des dispositifs innovants (dispositifs en ligne, fait par et pour les étudiants) mis en place ces derniers mois par l'université de Nîmes. 
En mars 2020, l’Organisation Mondiale de la Santé a déclaré la COVID-19 pandémie mondiale. En date du 16 mars 2020, le gouvernement français a annoncé un confinement national conduisant à une fermeture complète de l'ensemble des établissements scolaires et universitaires. Les universités ont alors, dans l'urgence, mis en place une continuité pédagogique basée sur l'enseignement à distance. Le 11 mai 2020 a débuté le premier plan de déconfinement dans l'ensemble du territoire français. Cependant, les universités françaises sont restées fermées aux étudiants, et cela jusqu'à la fin de l'année universitaire, conduisant à un recours massif aux évaluations à distance. En septembre 2020, les universités ont réouvert leurs portes aux étudiants, une reprise partielle des enseignements en présentiel se met alors en place au sein des campus universitaires, mais avec de nouvelles contraintes (p. ex., réduction du nombre d'étudiants accueillis, port du masque obligatoire). Cependant, dès le mois d'octobre, de nombreuses universités françaises ont refermé en raison d'un trop grand nombre de contaminations. Le 30 octobre 2020, le gouvernement français a annoncé un second confinement, imposant à nouveau aux universités le recours quasi-exclusif aux enseignements à distance, à l'exception de quelques enseignements pratiques autorisés par les rectorats. Le 15 décembre 2020 a débuté le second déconfinement. Cependant, ce n'est qu'à partir du mois de février 2021 que les universités françaises obtiennent l'autorisation d'accueillir de nouveaux les étudiants, mais avec des capacités d'accueil très limitées (20\% maximum des effectifs).

\section{Conséquences de la crise sanitaire sur la santé mentale des}

\section{étudiants}

La crise sanitaire de la COVID-19 a engendré de nombreuses contraintes chez les étudiants (p. ex., enseignements hybrides, examens à distance), venant s'ajouter à celles déjà présentes antérieurement (p. ex., stress des examens, éloignement familial). Cela n'a pas été sans conséquence sur leur santé mentale. En effet, toutes les études réalisées durant le premier confinement mettent en évidence une détérioration importante de la santé mentale des étudiants, s'illustrant notamment par une forte prévalence de symptômes anxio-dépressifs, que ce soit en France (Essadek \& Rabeyron, 2020; Husky et al., 2020; Le Vigouroux et al., 2021), ou dans d'autres pays à travers le monde (Kaparounaki et al., 2020; Odriozola-González et al., 2020). De plus, une étude française menée auprès de près de 3100 étudiants au cours du second 
confinement met en avant que 19,2\% des étudiants ont déclaré avoir eu des idées suicidaires au cours des douze derniers mois ${ }^{1}$.

Leur importante détresse psychologique peut en partie s'expliquer par le fait qu'avant la crise sanitaire les étudiants étaient déjà identifiés comme une population vulnérable (voir les revues de littérature de Paula et al., 2020; Stanley \& Manthorpe, 2001). Or, les personnes qui présentaient une détresse psychologique importante au préalable sont celles chez qui les conséquences psychologiques de la pandémie sont les plus fortes (Zhang et al., 2020). En outre, d'autres facteurs ont également été mis en évidence pour comprendre les conséquences délétères de la crise sanitaire sur la santé mentale des étudiants tels que leurs niveaux d'exposition aux médias (Wang et al., 2020), leurs changements de lieux de vie, la distance avec les membres de leur famille et/ou avec leurs amis (J. Lee, 2020; Sahu, 2020), et plus largement la solitude et l'isolement (Galea et al., 2020; Killgore et al., 2020).

Afin de mesurer l'état psychologique des étudiants français lors du premier confinement, nous avons conduit une étude (Le Vigouroux et al., 2021) auprès de 1297 étudiants provenant de différentes universités françaises (notamment l'université de Nîmes, l'Université de Lorraine, l'université de Strasbourg et l'Université Catholique de l'Ouest). Nos résultats montrent que 42,5\% des étudiants interrogés présentaient de possibles symptômes dépressifs (vs. 30\% avant le confinement: Boujut et al., 2009), et 33,4\% d'entre eux de possibles symptômes anxieux (vs. 24.2\% avant le confinement: Balayssac et al., 2018). Ces données viennent confirmer les données internationales sur le sujet. Par la suite, afin d'appréhender l'évolution de l'état psychologique des étudiants au cours du temps, nous avons réalisé une étude longitudinale en quatre temps de mesure : le premier durant le premier confinement (du 21 avril au 10 mai 2020), le second lors du déconfinement (du 9 juin au 27 juillet 2020), le troisième lors de la reprise des enseignements à l'université (du 12 au 27 octobre 2020) et le quatrième durant le second confinement (du 20 novembre au 11 décembre 2020). En premier lieu, nos résultats mettent en lumière que les symptômes dépressifs des étudiants Français sont particulièrement élevés pendant les deux périodes de confinement, comparativement aux périodes sans confinement. En d'autres termes, les symptômes dépressifs des étudiants apparaissent être étroitement liés au confinement, et semblent se résorber ensuite. Sur la base de ces résultats, nous pouvons supposer que la crise sanitaire actuelle pourrait ne pas avoir de

\footnotetext{
${ }^{1} \mathrm{https} / / / \mathrm{www}$.aefinfo.fr/depeche/643760-apres-une-enquete-sur-la-sante-mentale-de-ses-etudiants-1-upjvs-inquiete-du-trauma-provoque-par-la-crise-sanitaire
} 
conséquences à long terme sur les symptômes dépressifs des étudiants, contrairement à ce qui a été mis en évidence lors des précédentes pandémies (Bonanno et al., 2008; A. M. Lee et al., 2007; Liu et al., 2012). En second lieu, nos résultats mettent en évidence que : 1. les symptômes anxieux des étudiants sont particulièrement élevés pendant les deux périodes de confinement, 2. ils diminuent fortement lors du premier déconfinement, et 3. ils ré-augmentent à nouveau lors de la reprise des enseignements à l'université, malgré l'absence de confinement. En d'autres termes, les symptômes anxieux des étudiants restent particulièrement élevés même en l'absence de confinement, et cela notamment durant les périodes d'enseignement à l'université. Nous pouvons supposer que l'accumulation des contraintes académiques, qu'elles soient liées ou non à la COVID-19, a pu jouer un rôle important dans les symptômes anxieux des étudiants. Ainsi, la pandémie de la COVID-19 pourrait avoir des effets délétères à moyen, voire à long terme, sur le niveau d'anxiété des étudiants. Cette éventualité mériterait d'être au cœur des préoccupations des universités dans les mois à venir.

Avant même la crise sanitaire actuelle, l'accumulation des tracas quotidiens vécus par les étudiants (p. ex. la pression universitaire, les changements d'horaires, le cumul avec une activité professionnelle, les difficultés financières) a été identifiée comme jouant un rôle central dans la détérioration de leur santé mentale (Réveillère et al., 2001). Or, la pandémie de la COVID19 a obligé les étudiants à faire face à des nouveaux stresseurs (p.ex., passage des enseignements et des examens à distance), dans l'urgence et sans aucune préparation, mettant à l'épreuve leurs stratégies d'ajustement au stress (ou de coping). Les stratégies d'ajustement renvoient aux efforts cognitifs et comportementaux réalisés par l'individu pour s'adapter à une situation stressante. Généralement, certaines stratégies sont identifiées comme adaptées car celles-ci permettent de réduire efficacement le stress (p.ex., la réévaluation positive, c'est-àdire le fait de modifier l'interprétation d'une situation afin d'en identifier des conséquences positives). D'autres stratégies sont quant à elles identifiées comme inadaptées car elles ne permettraient pas de réduire efficacement le stress à long-terme (p.ex., le désengagement comportemental, c'est-à-dire l'évitement de certaines situations) (Muller \& Spitz, 2003). Dans le contexte spécifique de la pandémie de la COVID-19, des travaux de recherche réalisés durant le premier confinement ont mis en évidence que plus les étudiants mobilisaient des stratégies d'ajustement inadaptées (p. ex. l'évitement), plus ils présentaient des symptômes anxieux et dépressifs élevés (Le Vigouroux et al., 2021). Plus précisément, nous avons pu mettre en évidence que pour faire face aux évènements stressants liés au confinement, les étudiants utilisaient peu de stratégies d'ajustement au stress. Il est également important de relever qu'en 
moyenne les stratégies les plus utilisées par les étudiants sont des stratégies adaptées, et plus précisément les stratégies d'acceptation et de réévaluation positive (Le Vigouroux et al., 2021).

\section{Conséquences de la crise sanitaire sur le mode de vie des étudiants}

La pandémie de la COVID-19 n'a pas eu que des conséquences sur la santé mentale des étudiants, elle a également conduit à une modification importante de leur mode de vie. Déjà avant la crise sanitaire, les étudiants étaient identifiés comme une population présentant des modes de vie et des habitudes pouvant être défavorables pour leur santé, se traduisant notamment par des niveaux élevés de sédentarité, une activité physique faible (Castro et al., 2018, 2020) et un régime alimentaire déséquilibré (Deliens et al., 2016). Lors du confinement, la fermeture des universités et le recours massif aux enseignements à distance ont conduit à une majoration des comportements sédentaires des étudiants (Gallè et al., 2020; Gallo et al., 2020; Rodríguez-Larrad et al., 2021). Cela peut s'expliquer par une augmentation considérable de leur temps passé devant des écrans (Gallè et al., 2020), et une forte réduction de leurs déplacements (que ce soit les déplacements précédemment réalisés entre leur logement et leur lieu d'étude, ou encore ceux qu'ils effectuaient pour changer de salles entre chaque cours). En considérant les nombreuses conséquences délétères sur la santé psychologique et physique de l'inactivité et des comportements sédentaires (World Health Organization, 2020), il apparait indispensable que les universités engagent des actions visant à réduire la sédentarité des étudiants. En outre, la crise sanitaire a conduit à une modification de l'activité physique des étudiants. Gallo et al. (2020) mettent notamment en évidence une réduction importante de leur temps de marche. Cependant, il est aussi important de relever que certains auteurs ont démontré que lors du premier confinement, les étudiants ont pratiqué plus d'activité physique en semaine (Romero-Blanco et al., 2020) et ont augmenté la pratique d'activités de haute intensité (p.ex., cardio-fitness) ainsi que la pratique d'activités douces (p.ex., yoga) (Rodríguez-Larrad et al., 2021).

Nos données recueillies auprès d'étudiants français rendent également compte d'un niveau élevé de sédentarité chez les étudiants. Celui-ci apparait être particulièrement fort lors des périodes de confinement, mais il reste globalement élevé même au cours des périodes non confinées, ce qui peut s'expliquer par le maintien des enseignements à distance. Nous avons également pu mettre en lumière une modification du type d'activités physiques des étudiants durant le premier confinement, s'exprimant par une majoration de leur temps passé à réaliser des activités de faible intensité, et notamment des activités de type ménage, jardinage, ou encore 
bricolage. Néanmoins, nos résultats démontrent que les étudiants n'ont pas maintenu ces comportements actifs par la suite, que ce soit lors du déconfinement, de la reprise des enseignements à l'université, ou encore au cours du second confinement. En revanche nos résultats ne semblent pas mettre en évidence de modifications dans les comportements alimentaires des étudiants. Ces données laissent à penser qu'engager des actions visant la promotion des comportements actifs sont essentielles.

\section{Préconisations}

La pandémie de la COVID-19 a entrainé une révolution numérique dans l'enseignement supérieur (Strielkowski, 2020) permettant aux universités d'assurer une continuité pédagogique. Cependant, cela n'a pas été sans conséquence pour les étudiants. Certains se sont sentis dépassés, submergés par le flux d'informations, l'absence d'horaires réguliers, la réduction drastique des interactions sociales, ou encore par les obstacles numériques et techniques auxquels l'enseignement à distance les a confronté (Baticulon et al., 2020). Si nous disposons à ce jour de données probantes concernant les détériorations de la santé mentale et du mode de vie des étudiants, nous n'avons pas encore pu mesurer clairement les conséquences de la pandémie sur le risque de décrochage, d'échec universitaire, ainsi que sur les difficultés d'insertion professionnelle des étudiants pour l'année 2020-2021, voire au-delà. Il nous apparait donc essentiel que les universités augmentent et diversifient les dispositifs d'accompagnement psychologique et pédagogique, de prévention et de promotion de la santé, notamment en favorisant l'utilisation d'interventions innovantes. La crise sanitaire imposant une réduction des contacts sociaux, les interventions de prévention et de promotion de la santé en ligne (p.ex., page web, page Facebook, capsules vidéo ou audios) mériteraient d'être davantage utilisées, à l'instar de ce qui a été fait pour les enseignements. En outre, au vu de l'isolement ressenti par les étudiants, des interventions réalisées par et pour les étudiants nous semblent être à privilégier. Dans la continuité de ces préconisations, et sur la base des données obtenues dans nos différentes recherches, notre équipe a mis en place deux programmes d'intervention au sein de l’Université de Nîmes.

En premier lieu, un dispositif en ligne (dispositif Conf'Etu) centré sur le stress et l'apprentissage a été proposé aux étudiants de l'université de Nîmes, d'octobre à décembre 2020, et a été reconduit de mars à avril 2021. Ce dernier est composé de huit modules, à raison d'un module par semaine, organisés de la manière suivante : Introduction sur le stress (module 1), Comprendre l'apprentissage (module 2), Les stratégies d'évitement vs. d'acceptation face 
au stress (module 3), Les stratégies cognitives et métacognitives (module 4), Les stratégies de blâme de soi et de réévaluation positive face au stress (module 5), Motivation et activité physique (module 6), Faire face aux inquiétudes et aux incertitudes (module 7), Synthèse (module 8). Chaque module est constitué de deux vidéos, d'une durée maximale de 15 minutes, et contient des contenus diversifiés (p.ex., témoignages d'étudiants, informations, mise à disposition de ressources ou encore d'exercices). Les modules ont été le travail d'une coconstruction entre des enseignants-chercheurs et des étudiants. Plus précisément, la promotion des stratégies de réinterprétation positive et d'acceptation, et la réduction des stratégies d'évitement ont été privilégiées dans les modules dans le but de réduire leurs symptômes dépressifs. Dans le même ordre d'idée, des mises en garde concernant les stratégies de blâme de soi et l'expérimentation de nouvelles stratégies de gestion des inquiétudes leurs ont été proposées afin de réduire leurs symptômes anxieux. Enfin, des informations et des questionnements relatifs à la construction des apprentissages, aux stratégies d'apprentissages, à l'organisation du travail, ou encore au fonctionnement de la mémoire ont été dispensés dans différents modules. Nos résultats préliminaires mettent en évidence une réduction modérée et significative des symptômes anxieux, une réduction du sentiment d'impuissance apprise en lien avec l'université, ainsi qu'une augmentation de la croyance selon laquelle faire des erreurs est nécessaire à l'apprentissage chez les étudiants qui ont suivi le dispositif dans sa globalité.

En second lieu, un dispositif d'activités physiques en présentiel ayant pour but de favoriser le lien social et de promouvoir les comportements actifs a été porté par l'équipe pédagogique et les étudiants des formations STAPS de l'université de Nîmes (Mention Management du sport et Mention Activité Physique Adaptée - Santé), en collaboration avec les associations étudiantes. Chaque semaine, à raison de plusieurs créneaux par semaine, des activités d'aérobie / renforcement musculaire, ainsi que des activités douces (p.ex. yoga), sont proposées à l'ensemble des étudiants de l'université de Nîmes. Les séances sont limitées à 10 étudiants et sont préférentiellement réalisées en extérieur afin de respecter les gestes barrières et les recommandations ministérielles. Elles sont animées et organisées par les étudiants en STAPS, sous la supervision d'enseignants. Pour favoriser l'engagement des étudiants, ces activités sont organisées de manière ludique, notamment à travers la réalisation de sessions à thèmes (p.ex., Koh Lanta, chasse aux trésors...).

Enfin, depuis le $1^{\mathrm{er}}$ mars 2021, l'université de Nîmes a mis en place une plateforme d'appel à destination des étudiants (dispositif Gardons le contact). Douze étudiants tuteurs ont été recrutés avec pour mission principale de répondre à toutes les demandes formulées par les 
étudiants, que celles-ci soient liées à des difficultés psychologiques, financières ou encore numériques. En outre, ils ont également pour mission de téléphoner à l'ensemble des étudiants inscrit en Licence de l'université de Nîmes, afin d'évaluer leur état actuel. Pour ce faire, ils disposent d'un guide d'entretien basé sur les principaux domaines de la vie étudiante tels que leur assiduité en cours, leur motivation, leurs difficultés d'organisation, leur équipement matériel, leur logement, leurs finances, leurs activités physiques et sportives ou encore leur état psychologique. En fonction des difficultés rapportées, les tuteurs ont une fonction d'écoute et/ou d'orientation. Les principales orientations proposées sont synthétisées dans Figure 1. Les étudiants de l'université de Nîmes ont très favorablement accueilli cette initiative.

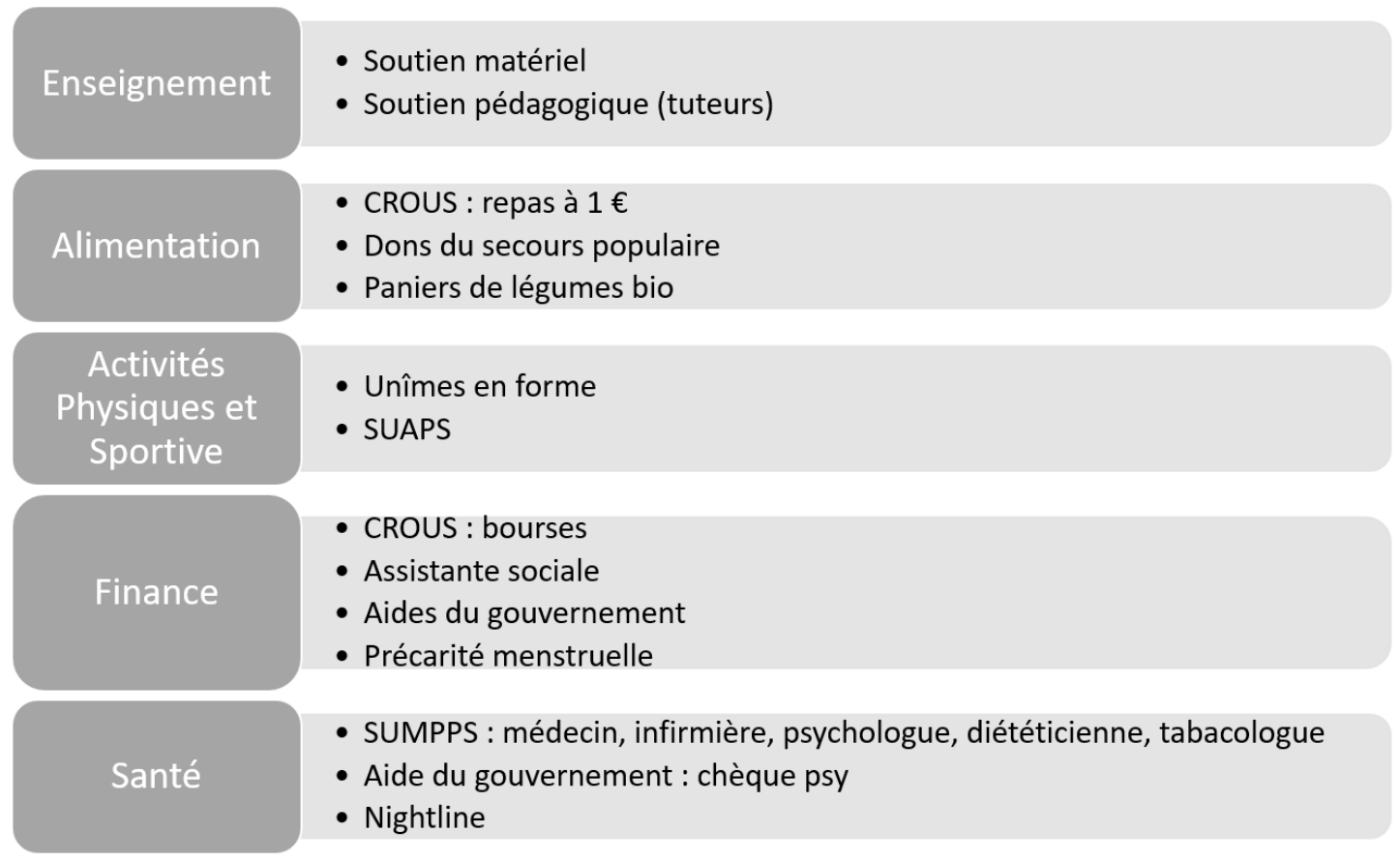

Figure 1. Ressources pouvant être proposée aux étudiants de l'université de Nîmes

\section{Conclusion}

En conclusion, toutes les données existantes rendent compte des multiples conséquences préjudiciables de la pandémie de la COVID-19 chez les étudiants, et de la nécessité de promouvoir des dispositifs innovants pour prévenir ces conséquences, notamment centrés sur la lutte contre la sédentarité, les gestions de l'apprentissage et des émotions. Les universités ont dû s'adapter dans l'urgence pour assurer une continuité pédagogique à leurs étudiants, mais sans toujours pouvoir pleinement prévenir les détériorations de santé des étudiants. Reste que de nombreux dispositifs et aides ont été mis en place par le gouvernement français, mais on peut déplorer la méconnaissance importante des étudiants vis-à-vis de ces derniers. Si la santé et le bien-être des étudiants est une politique prioritaire depuis une dizaine d'année dans les 
Presse Médicale Formation

pays anglo-saxons, ce n'est finalement que très récemment, et notamment depuis le début de cette crise sanitaire, que cela devient le cas en France. 
Balayssac, D., Pereira, B., Darfeuille, M., Cuq, P., Vernhet, L., Collin, A., Vennat, B., \& Authier, N. (2018). Use of psychotropic medications and illegal drugs, and related consequences among French pharmacy students - SCEP study: A nationwide crosssectional study. Frontiers in Pharmacology, 9(JUL), 1-12. https://doi.org/10.3389/fphar.2018.00725

Baticulon, R. E., Sy, J. J., Alberto, N. R. I., Baron, M. B. C., Mabulay, R. E. C., Rizada, L. G. T., Tiu, C. J. S., Clarion, C. A., \& Reyes, J. C. B. (2021). Barriers to Online Learning in the Time of COVID-19: A National Survey of Medical Students in the Philippines. Medical Science Educator, 2020.07.16.20155747. https://doi.org/10.1007/s40670-02101231-z

Bonanno, G. ., Ho, S. M. ., Wong, C. P. ., Chan, J. C. ., Kwong, R. S. ., Cheung, C. K. ., \& Wong, V. C. . (2008). Psychological Resilience and Dysfunction Among Hospitalized Survivors of the SARS Epidemic in Hong Kong: A Latent Class Approach. Health Psychology.

Boujut, E., Koleck, M., Bruchon-Schweitzer, M., \& Bourgeois, M. L. (2009). Mental health among students: A study among a cohort of freshmen. Annales Medico-Psychologiques, 167(9), 662-668. https://doi.org/10.1016/j.amp.2008.05.020

Castro, O., Bennie, J., Vergeer, I., Bosselut, G., \& Biddle, S. J. H. (2018). Correlates of sedentary behaviour in university students: A systematic review. In Preventive Medicine (Vol. 116, pp. 194-202). Academic Press Inc. https://doi.org/10.1016/j.ypmed.2018.09.016

Castro, O., Bennie, J., Vergeer, I., Bosselut, G., \& Biddle, S. J. H. (2020). How Sedentary Are University Students? A Systematic Review and Meta-Analysis. In Prevention Science (Vol. 21, Issue 3, pp. 332-343). Springer. https://doi.org/10.1007/s11121-020-01093-8

Deliens, T., Van Crombruggen, R., Verbruggen, S., De Bourdeaudhuij, I., Deforche, B., \& Clarys, P. (2016). Dietary interventions among university students: A systematic review. In Appetite (Vol. 105, pp. 14-26). Academic Press. https://doi.org/10.1016/j.appet.2016.05.003

Essadek, A., \& Rabeyron, T. (2020). Mental health of French students during the Covid-19 pandemic. Journal of Affective Disorders, 277, 392-393. https://doi.org/10.1016/j.jad.2020.08.042 
Galea, S., Merchant, R. M., \& Lurie, N. (2020). The mental health consequences of COVID19 and physical distancing: The need for prevention and early intervention. Depression and Anxiety, 180(6), 817-818. https://doi.org/10 .1002/da.20838

Gallè, F., Sabella, E. A., Ferracuti, S., De Giglio, O., Caggiano, G., Protano, C., Valeriani, F., Parisi, E. A., Valerio, G., Liguori, G., Montagna, M. T., Spica, V. R., Da Molin, G., Orsi, G. B., \& Napoli, C. (2020). Sedentary behaviors and physical activity of Italian undergraduate students during lockdown at the time of COVID-19 pandemic. International Journal of Environmental Research and Public Health, 17(17), 1-11. https://doi.org/10.3390/ijerph17176171

Gallo, L. A., Gallo, T. F., Young, S. L., Moritz, K. M., \& Akison, L. K. (2020). The Impact of Isolation Measures Due to COVID-19 on Energy Intake and Physical Activity Levels in $\begin{array}{lllll}\text { Australian } & \text { University } & \text { Students. } & \text { Nutrients, } & 12(6),\end{array}$ https://doi.org/10.3390/nu12061865

Husky, M. M., Kovess-Masfety, V., \& Swendsen, J. D. (2020). Stress and anxiety among university students in France during Covid-19 mandatory confinement. Comprehensive Psychiatry, 102, 152191. https://doi.org/10.1016/j.comppsych.2020.152191

Kaparounaki, C. K., Patsali, M. E., Mousa, D.-P. V, Papadopoulou, E. V. K., Papadopoulou, K. K. K., \& Fountoulakis, K. N. (2020). University students' mental health amidst the COVID-19 quarantine in Greece. Psychiatry Research, 290, 113111. https://doi.org/10.1016/j.psychres.2020.113111

Killgore, W. D. S., Cloonan, S. A., Taylor, E. C., \& Dailey, N. S. (2020). Loneliness: A signature mental health concern in the era of COVID-19. Psychiatry Research, 290(May), 113117. https://doi.org/10.1016/j.psychres.2020.113117

Le Vigouroux, S., Goncalves, A., \& Charbonnier, E. (2021). The Psychological Vulnerability of French University Students to the COVID-19 Confinement. Health Education \& Behavior. https://doi.org/10.1177/1090198120987128

Lee, A. M., Wong, J. G., McAlonan, G. M., Cheung, V., Cheung, C., Sham, P. C., Chu, C.-M., Wong, P.-C., Tsang, K. W. T., \& Chua, S. E. (2007). Stress and Psychological Distress among SARS Survivors 1 Year after the Outbreak. The Canadian Journal of Psychiatry, 52(4), 233-240. https://doi.org/10.1177/070674370705200405

Lee, J. (2020). Mental health effects of school closures during COVID-19. The Lancet Child \& 
Presse Médicale Formation

Adolescent Health, 4(6), 421. https://doi.org/10.1016/S2352-4642(20)30109-7

Liu, X., Kakade, M., Fuller, C. J., Fan, B., Fang, Y., Kong, J., Guan, Z., \& Wu, P. (2012). Depression after exposure to stressful events: lessons learned from the severe acute respiratory syndrome epidemic. Comprehensive Psychiatry, 53(1), 15-23. https://doi.org/10.1016/j.comppsych.2011.02.003

Muller, L., \& Spitz, E. (2003). Évaluation multidimensionnelle du coping: Validation du Brief COPE sur une population française. Encephale, 29(6 I), 507-518.

Odriozola-González, P., Planchuelo-Gómez, Á., Irurtia, M. J., \& de Luis-García, R. (2020). Psychological effects of the COVID-19 outbreak and lockdown among students and workers of a Spanish university. Psychiatry Research, 290, 113108. https://doi.org/10.1016/j.psychres.2020.113108

Paula, W. de, Breguez, G. S., Machado, E. L., \& Meireles, A. L. (2020). Prevalence of anxiety, depression, and suicidal ideation symptoms among university students: a systematic review. Brazilian Journal of Health Review, 3(4), 8739-8756. https://doi.org/10.34119/bjhrv3n4-119

Réveillère, C., Nandrino, J. ., Sailly, F., Mercier, C., \& Moreel, V. (2001). Étude des tracas quotidiens des étudiants : liens avec la santé perçue. Annales Médico-Psychologiques, Revue Psychiatrique, 159(6), 460-465. https://doi.org/10.1016/S0003-4487(01)00070-1

Rodríguez-Larrad, A., Mañas, A., Labayen, I., González-Gross, M., Espin, A., Aznar, S., Serrano-Sánchez, J. A., Vera-Garcia, F. J., González-Lamuño, D., Ara, I., Carrasco-Páez, L., Castro-Piñero, J., Gómez-Cabrera, M. C., Márquez, S., Tur, J. A., Gusi, N., Benito, P. J., Moliner-Urdiales, D., Ruiz, J. R., ... Irazusta, J. (2021). Impact of COVID-19 Confinement on Physical Activity and Sedentary Behaviour in Spanish University Students: Role of Gender. International Journal of Environmental Research and Public Health, 18(2). https://doi.org/10.3390/ijerph18020369

Romero-Blanco, C., Rodríguez-Almagro, J., Onieva-Zafra, M. D., Parra-Fernández, M. L., Prado-Laguna, M. D. C., \& Hernández-Martínez, A. (2020). Physical activity and sedentary lifestyle in university students: Changes during confinement due to the covid19 pandemic. International Journal of Environmental Research and Public Health, 17(18), 1-13. https://doi.org/10.3390/ijerph17186567

Sahu, P. (2020). Closure of Universities Due to Coronavirus Disease 2019 (COVID-19): Impact 
Presse Médicale Formation

on Education and Mental Health of Students and Academic Staff. Cureus. https://doi.org/10.7759/cureus.7541

Stanley, N., \& Manthorpe, J. (2001). Responding to students' mental health needs: Impermeable systems and diverse users. Journal of Mental Health, 10(1), 41-52.

Strielkowski, W. (2020). COVID-19 pandemic and the digital revolution in academia and higher education. Preprints. https://doi.org/10.20944/preprints202004.0290.v1

Wang, C., Pan, R., Wan, X., Tan, Y., Xu, L., Ho, C. S., \& Ho, R. C. (2020). Immediate psychological responses and associated factors during the initial stage of the 2019 coronavirus disease (COVID-19) epidemic among the general population in China. International Journal of Environmental Research and Public Health, 17, 1729. https://doi.org/10.3390/ijerph17051729

World Health Organization. (2020). WHO Guidelines on physical activity and sedentary behaviour. In World Health Organization. https://www.who.int/publications/i/item/9789240015128

Zhang, Y., Zhang, H., Ma, X., \& Di, Q. (2020). Mental health problems during the COVID-19 pandemics and the mitigation effects of exercise: A longitudinal study of college students in China. International Journal of Environmental Research and Public Health, 17(10). https://doi.org/10.3390/ijerph17103722 